\title{
Evolution of the Extragalactic Infrared-to-Ultraviolet Background and Gamma-Ray Attenuation
}

\author{
T. M. Kneiske and K. Mannheim
}

Sternwarte Göttingen, D-37083, Germany

\begin{abstract}
We describe a model for the evolution of the cosmic background radiation field from infrared to ultraviolet wavelengths tuned to observations of star formation and including the effects of reprocessing by dust and gas. With this model, we can compute the attenuation of gamma rays from extragalactic sources. The attenuation length for the vast majority of extragalactic gamma-ray sources (blazars and gammaray bursts at redshifts of order unity) depends on the evolving optical-toultraviolet background, whereas gamma-ray attenuation measurements of nearby sources such as Mrk 501 probe the infrared background. We focus on the cosmological aspects of the model and discuss the effects of changing cosmological parameters, star formation rate, initial mass function, and dust opacity on the resulting gamma-ray attenuation. A plausible choice of parameters leads to fair agreement between our model in the infrared and the observed attenuation of gamma rays from Mrk 501.
\end{abstract}

